\title{
Reparación endoscópica endonasal de fístulas de líquido cefalorraquídeo no quirúrgicas: Experiencia Hospital Clínico Universidad de Chile
}

\section{Transnasal endoscopic repair of nonsurgical cerebrospinal fluid leaks: Experience at the University of Chile Clinical Hospital}

\author{
Alfredo Abarca $A^{1}$, Javiera Pardo $\mathrm{J}^{2}$, Melchor Lemp $\mathbf{M}^{3}$, Carlos Stott $\mathrm{C}^{1}$.
}

\begin{abstract}
RESUMEN
Introducción: La fístula de líquido cefalorraquídeo (FLCR) no quirúrgica es una patología que requiere tratamiento quirúrgico en un alto porcentaje de los casos, siendo el abordaje endoscópico una buena alternativa.

objetivo: Describir la experiencia de nuestro hospital en el manejo endoscópico de esta patología.

Material y método: Mediante estudio retrospectivo descriptivo se analizan 11 casos clínicos. Se describen antecedentes demográficos, forma de presentación, estudio, etiología, técnica quirúrgica, seguimiento, tasa de éxito y complicaciones.

Resultados: Once pacientes, 9 de sexo femenino, edad promedio 44,5 años. Localización fosa anterior 8/11 y fosa media 3/11. El tamaño del defecto fue menor a 10 $\mathrm{mm}$ en todos los casos. La etiología fue traumática en 2/11, espontánea en 5/11 y congénita en 4/11, de éstas 3 por persistencia del conducto de Sternberg. El injerto fue mucosa más grasa 6/11 y mucosa sola en 5/11. La técnica fue overlay 8/11 y underlay en los 3 restantes. El injerto se colocó libre en 9/11 y pediculado en 2 casos. El seguimiento promedio fue 14 meses. Tasa de éxito de cierre de 100\% en la primera cirugía en todos los casos (11/11). No se evidenciaron complicaciones precoces ni tardías.

Conclusiones: La reparación endoscópica de FLCR no quirúrgica es una técnica poco agresiva, efectiva y de bajo riesgo.

Palabras clave: Fístula de líquido cefalorraquídeo, cirugía endoscópica, base de cráneo.
\end{abstract}

\section{ABSTRACT}

Introduction: The cerebrospinal fluid leak (CSFL) not surgical is a pathology that needs surgical treatment in a high percentage of the cases, being the endoscopic repair a good alternative.

\footnotetext{
Médico. Servicio de Otorrinolaringología, Hospital Clínico Universidad de Chile.

Médico Cirujano. Tesista de Magíster en Ciencias Biomédicas, mención Neurociencias, Universidad de Chile.

Médico. Servicio de Neurocirugía, Hospital Clínico Universidad de Chile.
} 
Aim: Describe the experience of our hospital in the endoscopic repair of this pathology.

Material and method: Retrospective descriptive study, 11 clinical cases are analyzed. There are described demographic antecedents, form of presentation, study, etiology, surgical technology, follow-up, rate of success and complications.

Results: Eleven patients, 9 females, average age 44,5 years. Anterior skull base defect 8/11 and medial skull base 3/11. The size of the defect was less than $10 \mathrm{~mm}$ in all cases. The etiology was traumatic in 2/11, spontaneous 5/11 and congenital in 4/11, these 2 was Sternberg ductus. The graft was mucosa plus fat $6 / 11$ and mucosa alone in $5 / 11$. The overlay thecnique was $8 / 11$ and underlay in 3 case. The graft was free on 9/11 and pedicle in 2 cases. Mean follow-up was 14 months. Success rate of close to $100 \%$ in the first surgery in all cases (11/11). There were no early or late complications.

Conclusions: Endoscopic repair CSFL not surgical is a slightly aggressive, effective and low risk technique.

Key words: Cerebrospinal fluid leak, endoscopic surgery, skull base.

\section{INTRODUCCIÓN}

Fístula de líquido cefalorraquídeo (FLCR) se define operacionalmente como la comunicación entre el espacio subaracnoídeo y la vía aerodigestiva superior. En base de cráneo la duramadre está firmemente adherida a la lámina cribiforme, techo etmoidal, y techo y pared lateral del seno esfenoidal. Cualquier proceso patológico localizado en estas estructuras puede crear una comunicación entre el espacio de líquido cefalorraquídeo y la cavidad nasal, consecuencia de la disrupción de aracnoides, duramadre, base de cráneo ósea y la mucosa. Las podemos clasificar, en primera instancia, según la fosa craneana de origen, en de fosa anterior y de fosa media. Dentro de las primeras, los principales sitios de formación de FLCR son el techo etmoidal, la lámina cribosa y la pared posterior del seno frontal; en las segundas encontramos el techo y pared lateral del seno esfenoidal ${ }^{1-4}$.

En cuanto a su etiología, las podemos clasificar en: quirúrgicas, traumáticas, congénitas y espontáneas. Las quirúrgicas son principalmente secundarias a cirugía de base de cráneo y cirugía endoscópica nasal. Las de origen traumático son generalmente secundarias a trauma craneano, cerrado o penetrante, con fracturas de base de cráneo y representan el $70 \%-80 \%$ de todas las FLCR. Las espontáneas aparecen generalmente secundarias a condiciones que aumenten la pre- sión ventricular, como hidrocéfalo congénito, infeccioso, postrauma 0 presencia de masas intracranealas ${ }^{1,3,5,6}$. Dentro de las espontáneas, se ha caracterizado un subgrupo con hipertensión endocraneana benigna, en el cual hay hallazgos clínicos y radiológicos de hipertensión endocraneana, pero sin causa aparente ${ }^{7-9}$. Entre las causas congénitas tenemos los meningoceles, meningoencefaloceles, encefalocele y la persistencia del conducto de Sternberg. Este último corresponde a la permanencia del conducto craneofaríngeo, que ocurre por falta de fusión de las diferentes partes del hueso esfenoidal durante la etapa embrionaria. Se convierte en un punto débil donde pueden aparecer encefaloceles $y / 0$ fístulas, por su ubicación lateral en el seno constituyen una zona de difícil abordaje quirúrgico (Figura 1) 10-15. $^{10}$.

La principal forma de presentación clínica es la rinorraquia intermitente que puede acompañarse de historia de trauma, cefalea o meningitis. El diagnóstico se confirma con la presencia de $\beta-2$ transferrina, teniendo el estudio bioquímico de LCR un valor limitado $0^{1,2,4}$. La localización preoperatoria de la FLCR se realiza principalmente con tomografía computarizada de alta resolución, lo que permite identificar el defecto óseo y planificar el abordaje quirúrgico. La tomografía computarizada con cisternografía es un estudio invasivo, que presta mayor utilidad cuando el defecto óseo es inaparente y la FLCR se encuentra activa. La resonancia magnética (RM) es un examen no invasivo, permite dife- 


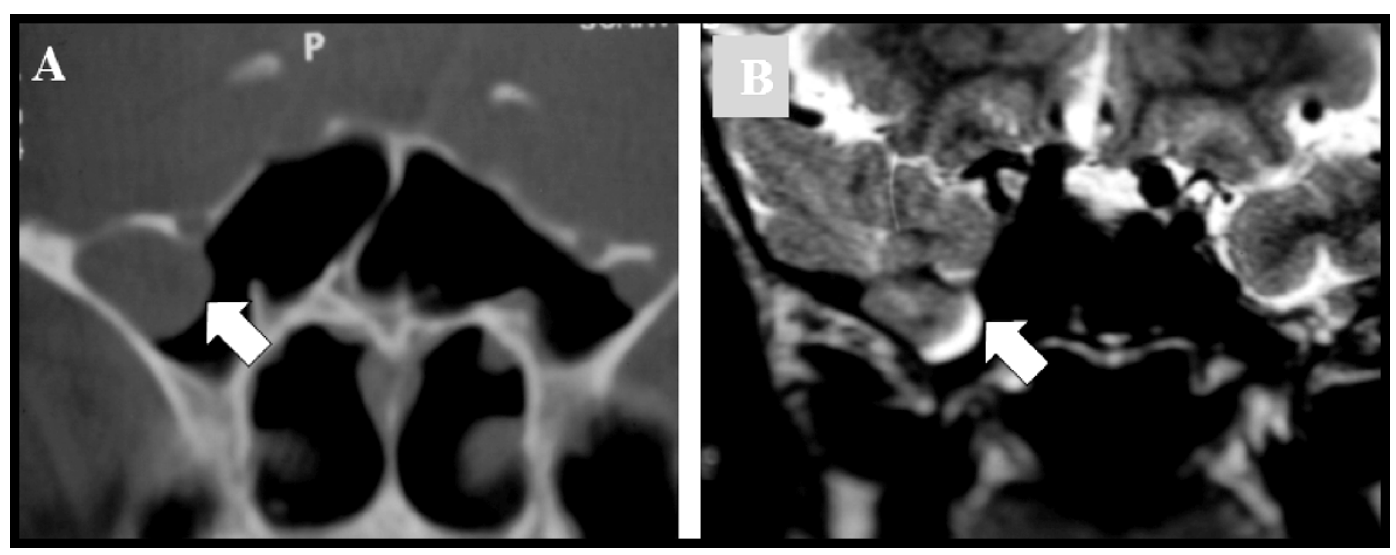

Figura 1. Paciente 10. Corte coronal a nivel del esfenoides. A) Tomografía computarizada. B) RM. Flecha: meningoencefalocele protruyendo a través de un conducto de Sternberg en la pared lateral del seno esfenoidal derecho.

renciar distintas densidades de tejidos blandos. En secuencia T2 pesada es posible visualizar el LCR saliendo por el defecto (Figura 2) ${ }^{16}$. La fluoresceína intratecal es usada principalmente en la localización intraoperatoria de la fístula ${ }^{1,17}$.

Dentro de las opciones de manejo se encuentran la conducta conservadora y el cierre quirúrgico. El manejo médico consiste en observación por

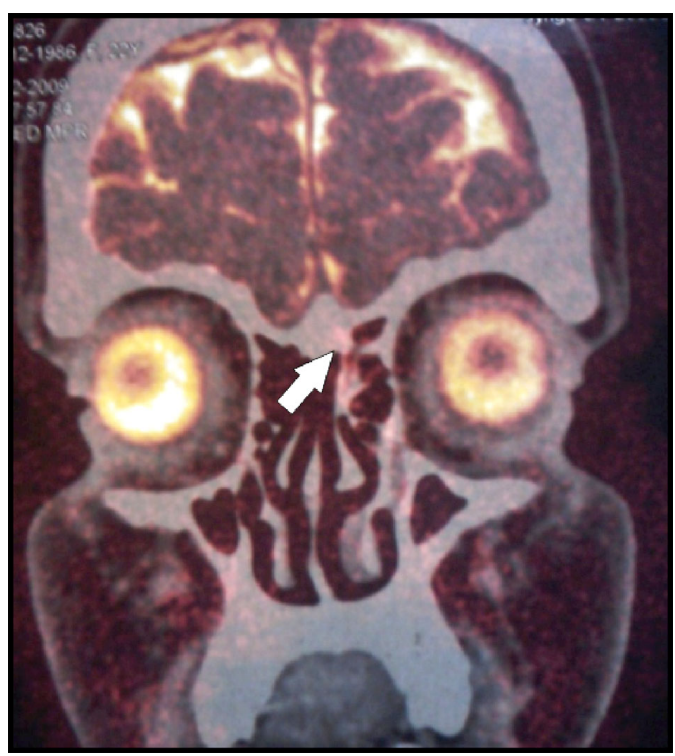

Figura 2. Paciente 8. Corte coronal de TC de cavidades paranasales, fusionada computacionalmente con RM en secuencia T2 pesada, se observa la salida de LCR a nivel del techo etmoidal izquierdo (flecha). un plazo de 7 a 10 días, esperando el cierre espontáneo de la fístula. El paciente se mantiene en reposo, con elevación de la cabeza, evitando maniobras de valsalva. El manejo con antibióticos es controversial, no siendo recomendado por la mayoría de los autores, ya que puede inducir resistencia microbiana. En los casos de hipertensión endocraneana, las medidas que ayudan a normalizar la presión intracraneana pueden facilitar el cierre espontáneo de la fístula. Entre estas medidas encontramos el uso de diuréticos como acetazolamida 0 la derivación ventriculoperitoneal2. Si el manejo conservador fracasa, está indicada la reparación quirúrgica.

Para una adecuada reparación quirúrgica hay que planificar el mejor abordaje, decisión multidisciplinaria, idealmente en equipo de base de cráneo, conformado por neurocirujanos, otorrinolaringólogos y radiólogos (Figura 3). Entre las opciones quirúrgicas tenemos el abordaje transcraneal, introducido por Dandy en 1926, el abordaje extracraneal, descrito por Dohlman en 1948, el abordaje transnasal microscópico, desarrollado por Hirsch en 1952. Finalmente tenemos el abordaje endoscópico, publicado por Wigand en 1981, 1,5,18,19. Desde su aparición en la década de los 80, la técnica de reparación endoscópica se ha masificado, siendo actualmente la primera elección en la mayoría de los casos. Presenta tasas de éxito en la primera cirugía sobre el $90 \%$ y cercana al $100 \%$ en la segunda intervención. Evita la craneotomía con tasas de 


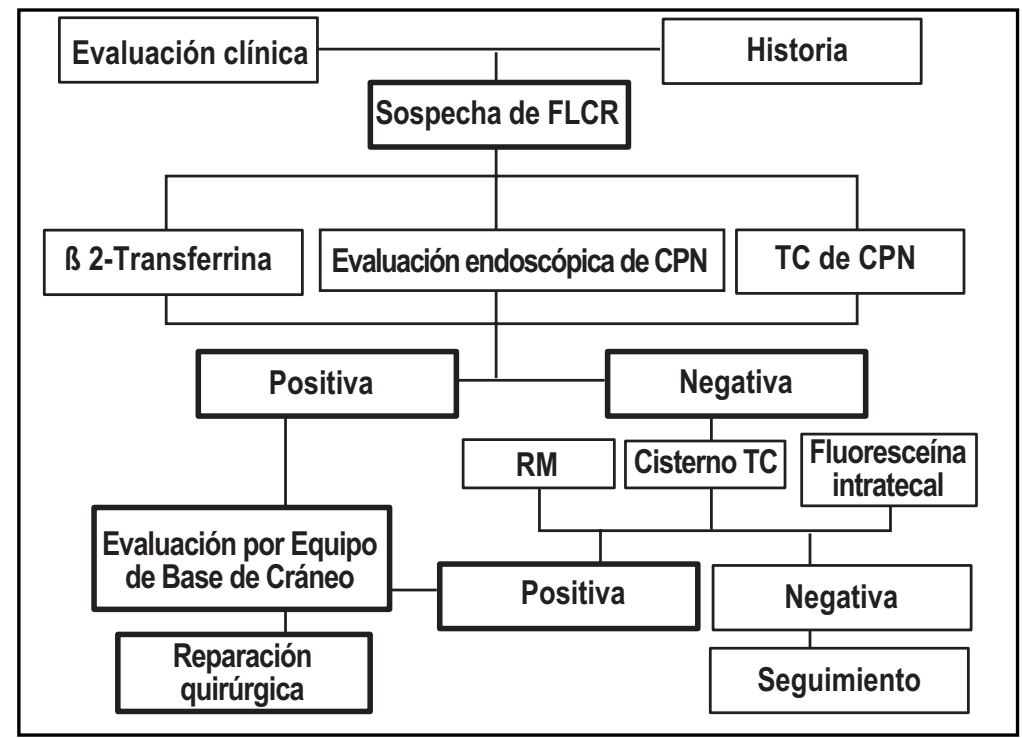

Figura 3. Algoritmo evaluación y manejo de FLCR.

complicaciones tan bajas como del 1\%1,18. En cuanto a la técnica utilizada para el cierre de la FLCR, es necesario definir el tipo de injerto a usar. Entre los cuales encontramos mucosa, grasa, fascia temporal, fascia lata, entre otros. Estos, a su vez, pueden colocarse como colgajos vascularizados o libres. Por otra lado, el posicionamiento del injerto puede ser underlay, es decir, en el espacio epidural, entre la duramadre y la base de cráneo ósea; o puede ser overlay, directamente sobre el defecto, externamente a la base de cráneo. Finalmente, una vez efectuada la reparación, el injerto debe ser fijado provisoriamente mediante el uso de adyuvantes, seguido de taponamiento anterior ${ }^{1,18,19}$.

El manejo posoperatorio requiere monitorización especializada en unidad neuroquirúrgica. La mayoría de los autores coinciden en el uso de antibióticos que crucen la barrera hematoencefálica en el perioperatorio. La medición y manejo de la presión intratecal, en casos de sospecha de hipertensión endocraneana, puede ir desde el uso de diuréticos hasta una derivación ventriculoperitoneal definitiva ${ }^{2,3,8,20}$.

El objetivo de este trabajo es describir el manejo de la FLCR de etiología no quirúrgica abordadas endoscópicamente vía transnasal en el Hospital Clínico de la Universidad de Chile.

\section{MATERIAL Y MÉTODO}

Estudio descriptivo retrospectivo. Se incluyen pacientes sometidos a reparación endoscópica de FLCR no quirúrgicas de base de cráneo, efectuadas entre septiembre de 2002 y abril de 2009. Fueron excluidos pacientes con FLCR de etiología quirúrgica, ya que tienen una forma de presentación y manejo diferente.

Se realiza revisión de historias clínicas, forma de presentación, estudio prequirúrgico, técnica quirúrgica, hallazgos intraoperatorios, manejo posquirúrgico, seguimiento, éxito de cierre y complicaciones.

\section{RESULTADOS}

Se analizaron un total de 11 pacientes, 9 de sexo femenino y 2 de sexo masculino. Con un promedio de edad de 44,5 años. El tiempo promedio que transcurrió entre el inicio de los síntomas y la cirugía fue de 15,2 meses (Tabla 1). En relación a la forma de presentación, todos los pacientes presentaban rinorraquia al momento del diagnóstico. El $64 \%$ (7/11) de los pacientes consultó por rinorraquia aislada, $27 \%$ (3/11) por rinorraquia más cefalea y $9 \%(1 / 11)$ presentó rinorraquia más meningitis (Figura 4). 
Tabla 1. Características demográficas y clínicas de los pacientes

\begin{tabular}{|ccccc|}
\hline Paciente & Género $(\mathrm{m} / \mathrm{f})$ & Edad & Inicio cuadro $^{*}$ & Seguimiento* $^{*}$ \\
\hline 1 & $\mathrm{~m}$ & 33 & 96 & 11 \\
2 & $\mathrm{f}$ & 39 & 24 & 1 \\
3 & $\mathrm{f}$ & 18 & 9 & 18 \\
4 & $\mathrm{f}$ & 40 & 3 & 12 \\
5 & $\mathrm{f}$ & 61 & 0,75 & 6 \\
6 & $\mathrm{~m}$ & 67 & 2 & 8 \\
7 & $\mathrm{f}$ & 22 & 1,5 & 10 \\
8 & $\mathrm{f}$ & 43 & 12 & 24 \\
9 & $\mathrm{f}$ & 58 & 8 & 24 \\
10 & $\mathrm{f}$ & 44 & 6 & 36 \\
11 & $\mathrm{f}$ & 44,5 & 5 & 14 \\
\hline
\end{tabular}

*Meses en relación a la cirugía.

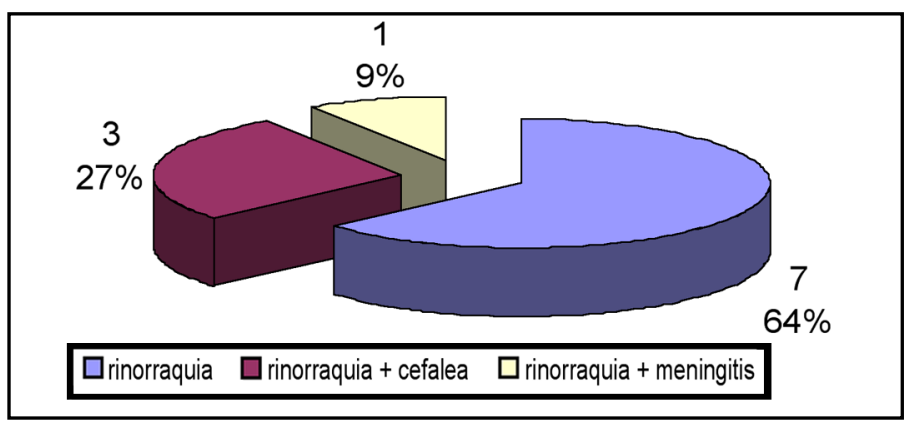

Figura 4. Formas de presentación de fístula de líquido cefalorraquídeo.

En relación al estudio prequirúrgico, 9/11 (82\%) pacientes fueron evaluados con $B 2$ transferrina y fluoresceína intratecal, ya sea preoperatorio 0 intraoperatorio. Un porcentaje algo menor fue evaluado además con examen físico-químico del líquido cefalorraquídeo (64\%). Todos los pacientes fueron evaluados con endoscopía nasal (11/11). El estudio imagenológico fue hecho con tomografía de cavidades paranasales en todos los pacientes. En algunos casos se realizó además resonancia (7/11) y tomografía computarizada de cerebro (8/11). Sólo en pocos casos se realizó cisternograma, ya sea mediante tomografía o radionucleótido (Tabla 2).

Con respecto a la etiología, se identificó $46 \%$ de fistulas espontáneas (5/11), 36\% de fistulas congénitas (4/11), de las cuales 3 son por persistencia del conducto de Sternberg. El $18 \%$ de las fístulas son de origen traumático (2/11) (Figura 5).
La localización de la FLCR fue en fosa anterior $8 / 11$ y en fosa media $3 / 11$. Del total de casos, $46 \%$ fue de techo etmoidal (5/11), y en el resto comprometía lámina cribosa 27\% (3/11) y esfenoides 27\% (3/11) (Figura 6). El tamaño promedio del defecto fue de $6 \mathrm{~mm}$, siendo en todos los casos menor a $10 \mathrm{~mm}$.

En todos los pacientes con reparación de fístula de fosa anterior, se realizó preparación con vasoconstrictor tópico, resección del cornete medio, etmoidectomía anterior y posterior, visualización del defecto y decolaje de la mucosa adyacente. En los pacientes con fístula de fosa media, se realizó además de lo anterior un abordaje transpterigoideo del segmento lateral del esfenoides, consistente en antrostomía maxilar, fresado de la pared posterior del seno, identificación y ligadura de la arteria esfenopalatina y seguimiento a través 
Tabla 2- Estudio preoperatorio

\begin{tabular}{|lrr|}
\hline & N/Total & $\%$ \\
\hline Laboratorio & & \\
B2 - transferrina & $9 / 11$ & 82 \\
Fluorescé́na intratecal (pre o intraoperatoria) & $9 / 11$ & 82 \\
Bioquímico LCR & $7 / 11$ & 64 \\
Endoscopía nasal & $11 / 11$ & 100 \\
Imagenología & & \\
TC cavidades paranasales & $11 / 11$ & 100 \\
TC cerebro & $8 / 11$ & 73 \\
RNM encefálica & $7 / 11$ & 64 \\
Cisternograma TC & $3 / 11$ & 27 \\
Cisternograma por radionucleótido & $1 / 11$ & 9 \\
\hline
\end{tabular}

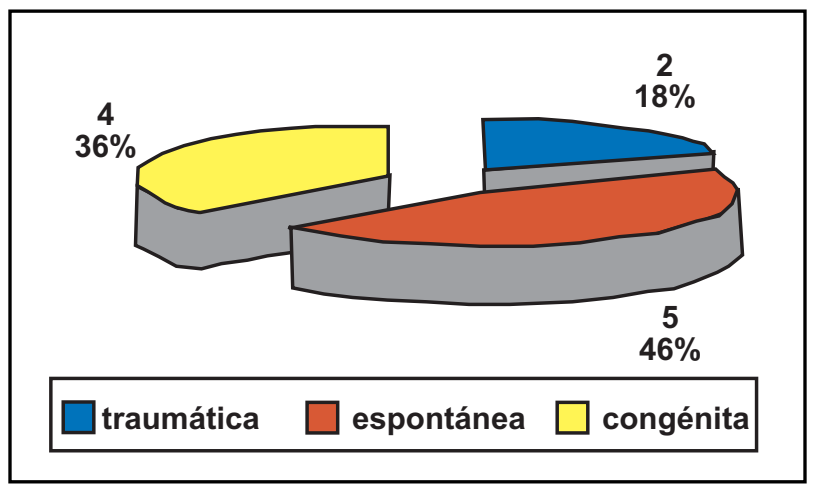

Figura 5. Etiologia de fístula de líquido cefalorraquídeo.

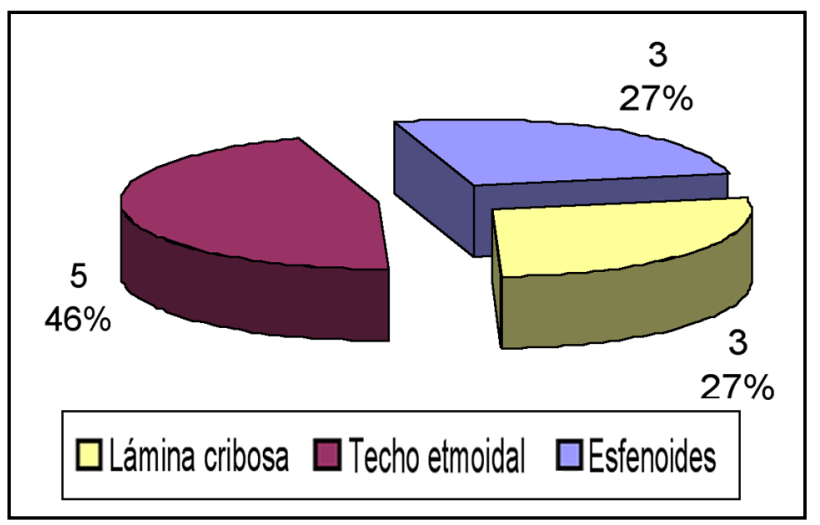

Figura 6. Localización de fístula de líquido cefalorraquídeo.

del canal del nervio vidiano hasta el esfenoides, con una esfenoidostomía ampliada.

Una vez expuesto el defecto, la reparación fue efectuada utilizando injerto de mucosa más grasa inguinal en $55 \%$ de los casos (6/11) y en el $45 \%$ restante (5/11) mucosa sola (Figura 7$)$. El injerto se posicionó overlay al defecto en 8/11 casos y underlay en los 3 restantes. En dos ocasiones se 


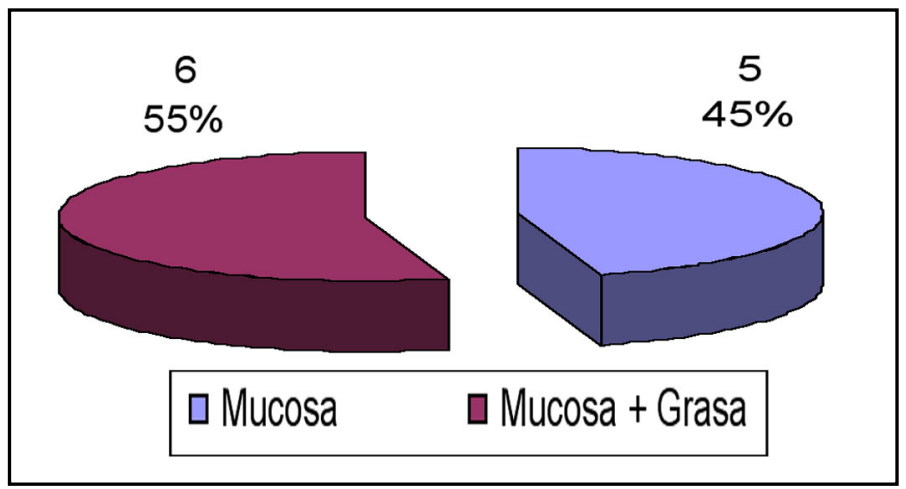

Figura 7. Tipo de injerto utilizado para reparación de la fístula de líquido cefalorraquídeo.

usó un colgajo pediculado y en el resto injerto libre. La mucosa utilizada fue de cornete medio en los 9 casos de injerto libre y de tabique nasal en los 2 casos de colgajo pediculado.

Luego de posicionado el injerto, se utilizaron materiales adyuvantes para asegurar la fijación de éste. En todos los pacientes se utilizó cola de fibrina, gelfoam $^{\circledR}$, siendo luego taponados anteriormente con meroce $^{\circledR}$. Sólo en dos pacientes se usó Surgice ${ }^{\circledR}$ y Duragel $^{\circledast}$ (Tabla 3).

Dentro del manejo posoperatorio, todos los pacientes fueron hospitalizados en unidades de intermedio quirúrgico, recibieron antibióticos endovenosos biasociados y acetazolamida oral. Sólo en 4 de los 11 pacientes se utilizó drenaje lumbar. Los pacientes completaron 6,8 días de hospitalización promedio (Tabla 4).

El seguimiento fue realizado por el equipo de otorrinolaringología en conjunto con neurocirugía, el cual consistió en evaluación clínica y endoscópica. El seguimiento promedio fue de 14 meses. Durante este seguimiento se evidenció una tasa de éxito del 100\% en el cierre de la FLCR, en la primera cirugía en todos los casos (11/11). Durante este tiempo no se evidenciaron complicaciones precoces ni tardías.

\section{DISCUSIÓN}

El total de los pacientes presentaron rinorraquia como síntoma de presentación, llama la atención el promedio de 15,2 meses transcurridos desde el inicio de los síntomas hasta la cirugía. Sin embargo, hay 2 pacientes que fueron derivados de un centro asistencial público que se alejan de la media, si se excluyen, el promedio baja a 5,25 meses. Uno de estos pacientes presentó 3 episodios de meningitis antes de la reparación.

Del estudio, destaca el alto porcentaje de pacientes (82\%) a los que se le practicó ß2transferrina, considerando que es un examen escasamente disponible en nuestro medio.
Tabla 3. Uso de adyuvantes en la reparación de FLCR

\begin{tabular}{|lcc|}
\hline Adyuvante & $\mathrm{N}^{\circ}$ de pacientes & $\%$ \\
\hline Cola de fibrina & 11 & 100 \\
Gelfoam $^{\circledR}$ & 11 & 100 \\
Merocel $^{\circledR}$ & 11 & 100 \\
Surgicel $^{\circledR}$ & 1 & 9 \\
Durage $^{\circledR}$ & 1 & 9 \\
\hline
\end{tabular}

Tabla 4. Manejo posquirúrgico

\begin{tabular}{|lcc|}
\hline Manejo & $\begin{array}{c}\mathrm{N}^{0} \text { de } \\
\text { pacientes }\end{array}$ & $\%$ \\
\hline Antibióticos & 11 & 100 \\
Acetazolamida & 11 & 100 \\
Drenaje lumbar & 4 & 36 \\
Promedio días de hospitalización & 6,8 & \\
\hline
\end{tabular}


Dada su alta especificidad y sensibilidad, nosotros proponemos su uso al comienzo del estudio del paciente con sospecha de FLCR. El estudio bioquímico del LCR se realizó en 7 de 11 pacientes, en la mayoría como aproximación inicial, pero su bajo rendimiento obliga a solicitar otros estudios, sólo lo recomendamos ante la imposibilidad de realizar B2-transferrina ${ }^{1,2}$. En nuestro trabajo, la fluoresceína intratecal fue usada en $9 / 11$ casos. Actualmente la utilizamos fundamentalmente en el intraoperatorio, siendo de gran utilidad en la localización del defecto y la comprobación del cierre de la fístula durante la cirugía ${ }^{1}$. No obstante su gran utilidad, su uso no está exento de riesgos. Se han descrito complicaciones como debilidad y parestesias de extremidades inferiores, convulsiones, opistótonos y déficit de pares craneanos ${ }^{20}$. Para prevenir estos efectos adversos, recomendamos administrarla siguiendo el protocolo de Stammberger ${ }^{21}$.

La totalidad de los pacientes tenía al momento de la cirugía una tomografía computarizada de cavidades paranasales, consideramos que este examen, al detallar la estructura ósea, es básico para planificar el abordaje. Además, en ocasiones, permite identificar el defecto óseo, asociado 0 no, a ocupación por densidad de partes blandas en el seno afectado. En 3 casos se realizó tomografía computarizada con cisternografía y en 1 caso con radioisótopos, ambos procedimientos invasivos, que requieren de inyección intratecal. En $7 / 11$ se realizó resonancia magnética, examen que en las modalidades dinámicas actuales, permite resaltar el LCR, siendo postulado por varios autores como una alternativa a métodos invasivos ${ }^{1,16,17}$ (Figura 3).

De la etiología de las fístulas destaca que $5 / 11$ son espontáneas, es decir, no se encontró causa aparente. De éstas, la totalidad eran pacientes de sexo femenino, con una edad promedio de 45,4 años, un índice de masa corporal promedio de $27 \mathrm{~kg} / \mathrm{m}^{2}$, sólo una refería cefalea. Hallazgos que nos obligan a pensar en el síndrome de hipertensión endocraneana benigna, ya que el manejo posoperatorio en estos pacientes debiese incluir monitorización de la presión intratecal y drenaje lumbar ${ }^{7-9,22}$.
El tamaño promedio del defecto fue pequeño ( 6 $\mathrm{mm}$ ) y en todos los casos menores a $1 \mathrm{~cm}$, esto pudiese ser explicable por la exclusión de las FLCR posquirúrgicas, las que suelen ser de mayor tamaño. En cuanto a la localización, la mayoría de las FLCR fueron de fosa anterior (8/11), y de éstas, 5 son del techo etmoidal. Destacan tres FLCR esfenoidales laterales, que obligó al equipo a realizar un abordaje transpterigoideo para lograr una buena exposición del segmento lateral del seno esfenoidal. Este abordaje resultó ser suficiente para lograr una adecuada reparación ${ }^{10-13}$.

En relación a la técnica quirúrgica para la reparación, destaca el uso de mucosa como injerto en todos los casos, en 6 de ellos asociado a grasa. Hegazy y col, en su metaanálisis de 14 publicaciones y un total de 289 FLCR, muestra que el injerto de mucosa es usada en el $50 \%$ de las reparaciones y la grasa en sólo el 19\%. Nosotros consideramos estos injertos de fácil obtención y escasa morbilidad en el sitio donante. Por otra parte, la técnica de colocación del injerto fue overlay en el $72 \%(8 / 11)$ de las FLCR. En la publicación de Hegazy su uso fue en el $62 \%$ de las reparaciones, elección que depende de múltiples factores, como el tamaño y localización del defecto, tensión sobre sitio afectado, entre otros. De los adyuvantes, destacamos el uso de cola de fibrina y gelfoam ${ }^{\circledR}$ en todos los casos, que en nuestra experiencia han resultado ser altamente útiles ${ }^{18}$.

Del posoperatorio es relevante que la estadía hospitalaria sea en promedio menor a una semana y sin complicaciones precoces, esto tomando en cuenta que la alternativa terapéutica es la craneotomía. El uso de antibióticos fue generalizado, ya que consideramos a la cavidad nasal un sitio contaminado. En nuestro trabajo, la indicación de drenaje lumbar no fue consensuada entre los médicos del equipo, siendo utilizada en 4/11 pacientes. A la luz de lo publicado recientemente en la literatura, actualmente nosotros recomendamos su uso sólo ante la sospecha de hipertensión endocraneana ${ }^{8,9}$.

El seguimiento promedio fue de 14 meses, tiempo en el cual no se evidenciaron complicaciones, y se constató el éxito en el cierre de la fístula en el $100 \%$ de los casos, en el primer intento 
quirúrgico. Hegazy muestra una tasa de éxito de $90 \%$ en la primera cirugía y $96 \%$ en el segundo intento, aunque incluyen causas quirúrgicas, que usualmente tienen defectos mayores y más complejos ${ }^{18}$.

\section{CONCLUSIÓN}

La experiencia en nuestro centro en el cierre endoscópico transnasal de las FLCR nos muestra que es una técnica poco agresiva, segura y efectiva. Donde el rol del otorrinolaringólogo es fundamental en el equipo de base de cráneo.

\section{BIBLIOGRAFÍA}

1. Locatelli D, Rampa F, Acchiardi I, et al. Endoscopic endonasal approaches for repair of cerebrospinal fluid leaks: nine-year experience. Neurosurgery 2006; 58 (suppl2): 246-57.

2. KeRR JT, CHu FW, BAYLES SW. Cerebrospinal fluid rhinorrhea: diagnosis and management. Otolaryngol Clin North Am 2005; 38(4): 597-611.

3. SChlosser RJ, Bolger WE. Endoscopic management of cerebrospinal fluid rhinorrhea. Otolaryngol Clin North Am 2006; 39(3): 523-38.

4. Martin T, LoehrL T. Endoscopic CSF leak repair. Current Opinion in Otolaryngology \& Head and Neck Surgery 2007; 15: 35-9.

5. Lee T, Huang C, Chuang C, Huang S. Transnasal Endoscopic Repair of Cerebrospinal Fluid Rhinorrhea and Skull Base Defect: Ten-Year Experience. Laryngoscope 2004; 114: 147581.

6. McMains K, Gross C, Kountakis S. Endoscopic Management of Cerebrospinal Fluid Rhinorrhea. Laryngoscope 2004; 114: 1833-7.

7. Woodworth B, Palmer J. Spontaneous cerebrospinal fluid leaks. Current Opinion in Otolaryngology \& Head and Neck Surgery 2009; 17: 59-65.

8. Carrau RL, Snyderman CH, Kassam ab. The management of cerebrospinal fluid leaks in patients at risk for high-pressure hydrocephalus. Laryngoscope 2005; 115(2): 205-12.

9. Basu D, Haughey BH, Hartman JM. Determinants of success in endoscopic cerebrospinal fluid leak repair. Otolaryngol Head Neck Surg 2006; 135(5): 769-73.

10. Castelnuovo P, Pagella F, Semino L, De Bernardi F, DeLÙ $G$. Endoscopic treatment of the isolated sphenoid sinus lesions. Eur Arch Otorhinolaryngol 2005; 262: 142-7.

11. Lal SY, Kennedy DW, Bolger WE. Sphenoid encephaloceles: disease management and Identification of lesions within the lateral recess of the sphenoid sinus. Laryngoscope 2002; 112: $1800-5$.

12. Tosun F, Carrau RL, Snyderman CH, Kassam A, Celin S, Schaitkin B. Endonasal endoscopic repair of cerebrospinal fluid leaks of the sphenoid sinus. Arch Otolaryngol Head Neck Surg 2003; 129(5): 576-80.

13. Al-Nashar IS, Carrau RL, Herrera A, Snyderman $\mathrm{CH}$. Endoscopic transnasal transpterygopalatine fossa approach to the lateral recess of the sphenoid sinus. Laryngoscope 2004; 114(3): 528-32.

14. Schick B, Brors D, Prescher A. Sternberg's canal - cause of congenital sphenoidal meningocele. Eur Arch Otorhinolaryngol 2000; 257: 430-2.

15. Castelnuovo P, Dallan I, Pistochini A, Battaglia P, Locatellı D, Bıgnamı M. Endonasal Endoscopic Repair of Sternberg's Canal Cerebrospinal Fluid Leaks. Laryngoscope 2007; 117: 345-9.

16. B Schuknecht, D Simmen, HR Briner, D Holzmann. Nontraumatic Skull Base Defects with Spontaneous CSF rhinorrhea and arachnoid herniation: Imaging Findings and Correlation With Endoscopic Sinus Surgery in 27 Patients. Am J Neuroradiol 2008; 29: 542-9.

17. Humbert Massegur Solench. Cirugía endoscópica avanzada de base de cráneo y espacios paranasales. Acta Otorrinolaringológica Española. October 2007. Vol. 58. Supplement 1.

18. Hegazy H, Carrau R, Snyderman, Kassam A, Zweig J. Transnasal Endoscopic Repair of Cerebrospinal Fluid Rhinorrhea: A Meta-Analysis. Laryngoscope 2000; 110: 1166-72.

19. Tosun F, Gonul E, Yetiser S, Gerek M. Analysis of different surgical approaches for the treatment of cerebrospinal fluid rhinorrhea. Minim Invasive Neurosurg 2005; 48(6): 355-60.

20. Keerl R, Weber RK, Draf W, et al. Use of sodium fluoresceina solution for detection of cerebrospinal fluid fistulas: analysis of 420 
administrations and reported complications in Europe and the United States. Laryngoscope 2004; 114: 266-72.

21. Stammberger $H$, Greistorfer K, Wolf $G$, LuXenberger W. Surgical occlusion of cerebrospinal fistulas of anterior skull base using intrathecal sodium fluorescein. Laryngorhinootologie 1997; 76: 595607.

22. Lopatin A, Kapitanov D, Potapov A. Endonasal endoscopic repair of spontaneous cerebrospinal fluids leacks. Laryngoscope 2007; 117: 345-9.

Dirección: Dr. Carlos Stott C.

Santos Dumont 999, Independencia

E mail: carlosstott@vtr.net 